Much has been written about " appendix" dyspepsia, " appendix " gastralgia, \&c., and it is common to see a patient who has confessed after the removal of his appendix that he has lost his " indigestion." This term may include almost, if not all, the symptoms of dyspepsia, even to those which were once regarded as pathognomonic of ulceration of the stomach, such as hæmatemesis. In some of these cases, when there has been no pain over the appendix region and no history of disease of the appendix, but where the pains have been epigastric, Dr. Blair Bell ${ }^{19}$ wrote :-

"If the patient complains of pain in the neighbourhood of the umbilicus, hypogastrium, or elsewhere, and that pain can be reproduced by pressure over the appendix, which may not be tender to pressure itself, then it will be found that the appendix is diseased."

During an operation for such a condition, exposure of the stomach will show that there is no evidence of disease in any part of it, "but its pyloric half is seen to be in vigorous and excited action." "The stomach becomes thick, contracted, and pale." 20

In the preceding section we have shown the "quiet" appendix responsible for varying forms of dyspepsia, and there can be little doubt that it is the cause of other morbid states. Cases of vomiting coming on at times without relation to food and without other symptoms such as pain, sometimes when the patient has gone to bed, sometimes at irregular intervals during the day may be cited.

Mr. W. H. B. Brook ${ }^{21}$ has described a case in which the removal of a chronic appendix cured a patient suffering from ovarian pain and menorrhagia. Sir Clifford Allbutt 22 published the account of a case which was regarded as "neurasthenia," "a weary aching, repining woman, a trouble to herself and all about her." Appendicectomy led to a permanent cure. An old history of peritonitis during adolescence was recalled afterwards. The possibility of a similar cause must be remembered in cases of this kind, for removal has been successful in other instances in which rest, \&c., had been only temporarily useful.

To these may be added cases of vague ill-health in which the patient looks toxic. Lambret, ${ }^{23}$ reported two cases, (1) ophthalmic migraine, which came on two years after an acute appendicitis; (2) epileptiform attacks which were of long standing and associated with much derangement of the digestive functions. Here there was a kind of aura originating in the cæcal region. No. 1 was cured; No. 2 much improved by removal of the appendix. Martley ${ }^{24}$ operated upon a man who had 12 attacks of Ménière's disease before an acute appendicitis with operation. Four years afterwards he had been free from further attacks. In another case an aural attack occurred at 37 and a second at 43 . Operation at 49 for acute appendicitis no further aural attack for 20 months.

Dr. Euzière considers that it is mostly in old-standing affections of the appendix, or at least of the digestive tract, that the transference of the pain in cases of early pneumonia is made to the abdomen. ${ }^{25}$

In a recent annotation in THE $\mathrm{LANCET}^{26}$ attention is drawn to a paper by Dr. O. Usland, which shows that in five cases of urinary symptoms due to bacilli treatment was not satisfactory until after removal of the appendix. In each case similar germs were found in the appendix, whence they had made their way to the right kidney. The end of the paragraph may be quoted, "The doctrine of focal infection has now reached a stage when it is liable to be parodied by undiscriminating enthusiasts and to fall into disrepute. But papers such as Dr. Usland's make it transparently clear that this doctrine contains more than a modicum of truth, and that it leads to therapeutic measures which would probably have been neglected without it."

20 Brit. Med. Jour., 1904, vol. ii., p. 1146.
20 Moynihan: Brit. Med. Jour., 1910, vol. i., p. 241.

Med. Jour., 1907, vol, ii, p. 70 22 Ibid., vol. i., p. 413

28 Arch. Prov. de Chir., October, 1911.

Brit. Med. Jour., 1914, vol. í., p. 1352

25 Rev. Franc. de Médec. et de Chir., 1909 THE LANCET, 1922, i., p. 807 .

\section{RETENTION OF URINE IN ELDERLY} MEN.*

BY J. SWIFT JOLY, F.R.C.S. ENG.,

SURGEON TO ST. PETER'S HOSPITAL FOR STONE AND TO THE LONDON LOCK HOSPITAL : CONSULTING UROLORIST, WANDSWORTH MUNICIPAL HOSPITAL.

As senile enlargement of the prostate is the most common cause of retention of urine in men over 60 years of age, I shall only deal with the immediate treatment of prostatic obstruction.

\section{Symptoms of Prostatic Obstruction.}

As the prostate becomes enlarged, the first evidence of retention is the presence of residual urine. This means that the patient never empties his bladder completely, but always leaves a certain residue behind. In the earlier stages of the disease the patient is quite unaware of this condition, and expresses great surprise when it is demonstrated to him. The amount of residual urine may vary from a few drachms up to a couple of pints, but from a clinical point of view it is better to divide these patients into two classes, according as the amount of residual urine is small or large. I have selected 10 ounces as an arbitary limit, and will speak of patients with less than 10 ounces residual as cases with a small amount of residual urine, and patient who retain more than half a pint as cases with a large amount of residual urine.

A patient with a small amount of residual urine has increased frequency of micturition, especially at night. He must rise from two to four or fire times during the night to pass urine. During the day the frequency is not so marked, and he can retain urine comfortably for two or three hours. The stream is diminished in volume, and the force of the jet is poor. There is often some slight delay in commencing the act of micturition. Beyond this he has little or no inconvenience, as no pain is felt unless infection or a stome is present. These patients are seldom liable to complete retention, and if it does occur it is only transitory, and spontaneous micturition is re-established after two or three days. Retention is usually caused by errors in eating or drinking especially by over-indulgence in alcohol, by a chil or wetting, by constipation, or if the patient has been forced to hold his urine for a considerable time after the desire to micturate has been experienced. If, however, his bladder is infected the frequency is more marked, and is diurnal as well as nocturnal, and pain or scalding along the urethra is felt during the act. Infection always renders the patient more liable to retention.

A patient with a large amount of residual urine has marked frequency of micturition. He is compelled to pass urine from five to ten times at night, and about every hour or oftener during the day. The stream is little more than a dribble, and there is no force in the jet. There is a long latent period, and he often has to wait for several minutes before the urine commences to flow. There is also a consider able amount of difficulty, and involuntary straining, which is sufficient to bring on an "attack of piles." However, the patient soon realises that straining does not help him to empty his bladder. There is always polyuria, the amount excreted in the 24 hours being increased to 80 or 100 ounces, or even more. I have recently seen a patient in this condition who had been passing 12 pints of urine a day for several weeks. The urine is pale, has a very low specific gravity1005 to 1010 and usually contains a trace of albumin. The percentage of urea and of other urinary solids is notably diminished. There is little or no pain, except a slight suprapubic ache, which is due to distension of the bladder, and sometimes a dull pain in the loins due to back-pressure on the kidneys. In addition to these local signs, the patient generally shows definite evidence of renal failure. The clinical

A post-graduate lecture delivered at the Wandsworth Municipal Hospital. 
signs of renal failure are loss of woight, progressive weakness, headaches, thirst, dryness of the mouth and throat, a dry glazed tongue, distaste for solid food, constipation, a dry, harsh skin, and a peculiar earthy pallor. Some of these signs are usually present, and should always be carefully looked for, as they are danger signals, and indicate that prompt measures must be taken or the patient will lose his life.

The next stage in prostatic obstruction is complete and chronic retention, or over-distention with overflow incontinence. The latter is the most advanced stage of uncomplicated prostatic obstruction, and therefore the most dangerous for the patient. A patient with overflow incontinence complains that he first noticed the urine escaping during sleep, but later on the incontinence was also diurnal. He gives a history of frequency of micturition extending over several years, and may be unaware that there is a large accumulation in his bladder. He states that he has been failing both mentally and physically for some time, and invariably presents some signs of renal failure. On examination of the abdomen a large pear-shaped swelling is noticed. It commences behind the pubis, and extends upwards to, or even above, the umbilicus. It exactly resembles the uterus of about the sixth month of gestation in size, shape, and appearance. It is painless, but on pressure there is a desire to micturate. It can be easily palpated, because the abdominal walls are lax; in cases of acute retention it is often difficult to palpate the bladder on account of rigidity of the recti. It is dull on percussion. When an over-distended bladder is once seen the clinical picture is so marked that it can never be forgotten. These patients are always free from infection. The urine is clear and pale, the specific gravity is usually below 1005, and there is generally a trace of albumin. 'The percentage of urea and other urinary solids is very low. There is always marked polyuria. These patients are extremely prone to infection unless rigid asepsis is maintained during catheterisation, and if they once become infected they invariably die within a few days.

From its earliest stage prostatic obstruction exerts an injurious effect on the kidneys. It is a well-known fact that in the later stages the ureters and the renal pelves become dilated, and the true renal tissue atrophied by pressure. It is often difficult to see any renal cortex at all at a post-mortem examination of a patient who has died as a result of prostatic obstruction, and the microscope reveals the fact that the small amount of excretory tissue left is almost strangled by fibrous tissue. But it is not so well recognised that this gradual destruction commences almost in the earliest stage of the disease. A man with an unrelieved prostatic obstruction is constantly damaging his kidneys, and may be compared to a man living on his capital. If the capital is sufficient, and he draws on it slowly, all may go well, but if the capital is insufficient to meet the demands put upon it, he will some day be penniless. The prostatic case may live for a considerable time in apparently good health, but his renal tissue is steadily being consumed. If a crisis occurs he suddenly becomes uræmic. A slight amount of renal failure does not give rise to any clinical signs, and can only be detected by means of modern tests of the renal function, and patients who show the slightest clinical signs of renal failure must have badly damaged kidneys. For this reason the apparently healthy condition of many of these prostatic cases is most misleading.

The Treatment of Prostatic Obstruction.

The only effectual treatment of prostatic obstruction is removal of the gland, and the question of operation should be carefully considered unless some definite contra-indication exists, but there are very few contra-indications to prostatectomy. However, I now wish to point out the best methods of dealing with some of the acute accidents that may occur in the course of chronic prostatic obstruction.
In acule retention it is perfectly easy to pass a cathoter and empty tho hadder, but this may be the very worst thing for the pationt. Bofore catheterising one should examine the abdomen, and percuss out the area of bladder dullness. If this does not rise more than two inclies above the pubis there is lithle risk in emptying the bladder completely, but even in this case the patient should immediately be sent home to bed. He should be told that it will be necessary to catheterise him again in six or eight hours, and that during the interval he should have a brisk purge, and take plenty of fluids. Hexamine, or some other urinary antiseptic, should be prescribed as a prophylactic. The patient should be kept in bed for at least a week, or until the power of spontaneous micturition returns. It is not usual for these patients to pass at once into the stage of complete catheter life, as the attack of retention usually only lasts about four or five days, but it should be looked on as a warning, and a foretaste of a condition that will become permanent if the obstruction is allowed to persist.

If, however, it is found that the bladder is overdistended and either forms a palpable turnour in the abdomen or else gives a dull percussion note rising higher than two inches above the pubis, it is extremely dangerous to empty the bladder completely when the patient is first seen. If he is in great distress a catheter may be passed, but not more than 15 ounces of urine should be drawn off. This amount is the maximum that should ever be drained from an overdistended bladder under these circumstances, and the withdrawal of this amount will be quite sufficient to relieve the patient's distress. After the patient has been put to bed his bladder can be emptied slowly by either of two methods. The first is by regular catheterisation every six or eight hours, withdrawing a slightly larger quantity of urine every time the instrument is passed. For instance, if 15 ounces is withdrawn on the first occasion 20 may be taken away on the second, and 25 on the third, and so on until the bladder has been completely emptied. Once this stage is reached it is most important not to allow the bladder to refill, and a course of regular catheterisation, at least three times in the 24 hours, should be instituted. If the power of spontaneous micturition returns a catheter should still be passed every morning and evening, unless the amount of residual urine falls to less than 10 ounces. The second method of emptying an over-distended bladder is to pass a catheter, previously fitted with a spigot so that the urine cannot escape, and to tie it in place. The spigot should be removed at the end of every hour, and from 4 to 5 ounces of urine drained off. It is most important that the spigot should not become loose and allow all the urine to escape. If it does, the bladder should immediately be filled up with sterile normal saline, or boric lotion. The most important points in this treatment are: (1) never empty an over-distended bladder in an old man suddenly, and (2) once such a bladder is emptied, never allow it to become filled again.

A patient with chronic distension and overflow incontinence is in a still more critical condition, but he rarely suffers pain. His only complaint is the inconvenience and annoyance of the urine dribbling away. It would be better for him if he did suffer, as then he would not allow the obstruction to become so advanced before seeking advice. It is advisable not to atterapt catheterisation on such a patient in one's surgery or in an out-patient department. He should be put to bed before any instrument is passed. If a fair amount of urine appears to be dribbling away it is better not to pass a catheter at once. Let him drain into a urinal, and estimate the amount of urine passed in the 24 hours. Suppose, for example, 96 ounces were passed in this time, the average hourly excretion is 4 ounces. Then pass a catheter, armed with a spigot, and draw olf 5 ounces every hour. By this means the accumulation in the bladder is diminished by one ounce an hour. It is D 2 
essential that the bladder should be emptied very slowly, and the pressure only allowed to fall very gradually. A rough estimate of the time necessary to empty one of these over-distended bladders can be made by measuring the height to which it rises above the pubis. The height of the vesical swelling should not be reduced more rapidly than one inch per diem, so that if the bladder rises four inches above the pubis one should take four days to empty it completely. The reason I lay such stress upon slow drainage is that if the bladder is suddenly emptied there will be a sharp attack of hæmaturia followed by suppression of urine, and the greatest difficulty will be experienced in getting the kidneys to act again. As a rule, the patient dies about five or six days after his bladder has been emptied too rapidly. The reason for this suppression is as follows: the sudden relief of the urinary pressure causes an intense engorgement of the renal venules, which have lost their tone, and this venous engorgement may be sufficient to impede the renal circulation so much that the excretion of urine ceases. The treatment is to re-establish the urinary pressure by filling up the bladder with sterile fluid. When the excretion of urine commences again the bladder should be emptied so slowly that the veins are given sufficient time to recover their tone before the urinary pressure falls to zero. Once a chronically overdistended bladder has been emptied regular catheterisation must be instituted.

I do not recommend catheter life for prostatic retention in any case. It should never be recommended as a permanent condition. In the first place, it is dangerous. The mortality during the first month of catheter life is 9 per cent. This is greater than the operative mortality after prostatectomy. In the second place, it is a miserable existence. The majority of patients who have tried it sooner or later come to have the gland removed. Self-catheterisation may make the downward path less steep, but it does not make it any smoother or less dangerous. Lastly, I have never seen a patient who was able to avoid infection. If he is lucky the infection remains localised to the bladder, but usually it sooner or later spreads up to the kidneys. Any patient suffering from enlargement of the prostate who has more than 2 ounces of residual urine should be advised to have the gland removed without delay. When it is done in this stage the operation is a prophylactic measure, and acts as an insurance against future trouble.

\section{Technique of Catheterisation.}

Choice of Instrument.-For ordinary use a gumelastic coude catheter is the best. It is almost the only type of catheter used at St. Peter's Hospital. The objection that gum-elastic instruments cannot be boiled no longer holds good. Modern instruments will stand boiling if certain precautions are observed. The catheter should be dropped into boiling water and left there for two minutes, and theil cooled in a dilute antiseptic lotion. It is important that the catheter should not touch either the bottom or the sides of the steriliser, as they are always hotter than the boiling water, and their temperature is sufficient to destroy the varnish. This means that the steriliser should be long enough to take the catheter without bending it, and if there is no tray in the steriliser the catheter should be wrapped up in a piece of lint. It should also be remembered that when the catheter is in the boiling water the varnish is extremely soft, and liable to damage if roughly handled. After use the catheter should be washed inside and out in cold water, and put away dry.

Lubricant.-Sterilised olive oil is the best lubricant as it is the best medium for diminishing friction, but it is difficult to prevent it from becoming contaminated, and it offers no resistance to the growth of organisms. Liquid paraffin is an excellent lubricant, and does not suffer from this objection. It is best to add one part of paraffin to ten of water, and boil the whole before use. The only objection to this lubricant is that it is inconvenient if the surgeon is called out to see a patient in his own home. In this case I prefer one of the catheter jellies that are sold in collapsible tubes. They are composed of a mucilage of gum, to which an antiseptic such as oxycyanide of mercury is added. This gives fair lubrication, and has the advantage that it is solub] in water, and therefore can be used for cystoscopy.

Asepsis.-Once the catheter has been sterilised one should not touch any part of the instrument that will enter the patient's urcthra. This is only possible if either a gum-elastic or a metal instrument is used. The great drawback to soft rubber catheters is that this method of introduction is impossible. A soft rubber catheter must be very thoroughly lubricated and must be passed inch by inch. Any organisms on the surgeon's hands are liable to be carried by the lubricant into the bladder. On the other hand, a gum-elastic catheter has sufficient backbone, when it is at the ordinary temperature, to allow its being passed while held by its extreme end. The portion within the urethra soon reaches the body temperature, and is then soft enough to follow the curve of the urethra without doing any damage. The patient's prepuce should be fully retracted, and the whole penis thoroughly washed, first with soap and water and then with an antiseptic, and the terminal portion of the urethra should be syringed out with boric lotion, or oxycyanide of mercury ( 1 in 6000 ).

Technique of Passing a Catheter.-Stand on the right side of the patient. Hold the penis behind the corona between the middle and third fingers of the left hand, while the lips of the meatus are held open by the thumb and index finger. Hold the catheter by its proximal end in the right hand, and insert the tip into the meatus. The coudé beak should always press against the roof of the urethra. The penis should be pulled upwards by the left hand, the action being much more that of pulling the penis over the catheter than pushing the instrument down the urethra. If the penis is kept on the stretch the cul-de-sac of the bulb will be obliterated, and the coudé point will automatically enter the membranous urethra. In most cases it slides easily into the bladder, but if the middle lobe of the prostate is very large it may prevent the onward passage of the instrument. If this happens, withdraw it about half an inch, rotate it slightly, and then pass it forward again. It will then most likely travel up in the groove between the middle and lateral lobes. Usually no difficulty will be experienced if a large (Nos. 10 to 12 E.) catheter is used, and all the movements are carried out slowly and gently, and if the patient does not strain or resist. If a soft instrument cannot be passed, a coudé catheter mounted on a stylet should be tried. A 1/4 Lister's sound makes the best stylet, but as it is usually too short for the ordinary catheters they must be cut down to fit it. Grasp the penis as before, but hold the instrument, point downwards, horizontally over the middle line of the patient's abdomen. Insert the tip into the meatus, and pull the penis upwards over the catheter. Keep the instrument flat along the patient's abdomen till its point reaches the bulb, then raise the handle, and it will at once slip into the posterior urethra. Keep the penis on the stretch the whole time, and by depressing the handle between the patient's thighs the bladder will be reached. If the catheter catches, withdraw it about half an inch, then holding the stylet steady, gradually push the catheter forward over it. This will cause the point to curve forwards, and slip over the projection of the middle lobe. The greatest care and gentleness is necessary in all these manceuvres, as the enlarged prostate is very soft, and it is extremely easy to make a false passage. If any difficulty has been experienced in passing the instrument it is advisable to tie it in place for 24 hours. This will have the effect of straightening out the urethra, and will render subsequent catheterisation easy. If a blood-clot blocks the eye of the 
instrument the best method of freeing it is to inject a small quantity of lotion (about balf an ounce) forcibly into the bladder by means of a syringe. Lastly, if all attempts at catheterisation fail the bladder must be punctured above the pubis. It is best to cut down on the bladder, make an opening large enough to admit the tip of the index finger, and fix a de Pezzer self-retaining catheter in it. The urine can then be drained off slowly through it. If there are no facilities at hand for an open operation temporary relief may be given by puncturing the bladder by means of a trocar and cannula, but immediate steps should be taken to move the patient into a nursing home or hospital for a cystotomy.

\section{PERI-ARTERIAL INJECTION OF ALCOHOL IN THE TREATMENT OF SENILE GANGRENE.}

(Preliminary Communication.)

BY W. SAMPSON HANDLEY, M.D., M.S. LOND., F.R.C.S. ENG.,

SURGEON TO THE MIDDLESEX HOSPITAL.

THE two following cases seem to be worthy of detailed record as illustrating a new method of producing vaso-constrictor paralysis, a method free from some of the objections attendant on the operation of sympathectomy introduced by Leriche, of Lyons.

CASE 1.-Female, aged 69, was admitted to the Middlesex Hospital under my care on Dec. 26th, 1921, for senile gangrene of the right foot. Fifteen years previously the patient suffered an amputation through the right forearm for "blood poisoning." This doubtless arose from a septic infection of a finger. The stump is healthy, but the illness no doubt did toxic damage to her arteries. Two years before admission Mr. T. H. Kellock removed half her tongue for carcinoma. There is no recurrence. Ten days ago she noticed her right foot felt cold and numb. It was tender and she could not comfortably put it to the ground. She noticed, too, that it was purple and discoloured.

Condition on Admission. - A thin, rather frail woman, looking quite her age. All the toes of the right foot are blackish, cold, wrinkled, and almost immobile. Some variations in colour are recognisable. While the fourth and fifth toes are almost black, the great toe and the second and third toes are dark purple in colour. The gangrene appeared to extend some distance along the metatarsals both on the dorsal and plantar aspects. On the dorsum the dark-purple discoloration of the skin, accompanied by loss of natural heat, occupied a triangular area with its apex directed backwards reaching to the base of the second metatarsal. The boundary of this area crossed the neck of the first and the head of the fifth metatarsal, and showed traces of an incipient line of demarcation. On the sole the dark discoloration of the skin extended a little farther back, and was more sharply triangular. It was mottled with patches of lighter purple, and the loss of natural heat was less complete. Tactile sensation was completely lost over the toes and over the purple area of the foot. The patient complained only of slight twinges of pain in the foot. No pulse could be felt in any of the arteries of the right lower limb, even in the femoral. The femoral and popliteal arteries were hard, cord-like, and pulseless. In the left femoral artery pulsation was present, but the left popliteal and its branches were pulseless. On this side also the arteries were hard and cord-like, and the left foot was blue and cold, though distinctly warmer to the hand than the portion of the right foot above the gangrenous area. The urine was free from sugar and albumin.

Course of Case.-During the first three weeks of January the gangrenous area remained dry and the temperature did not rise above $99^{\circ} \mathrm{F}$. The discoloration of the foot became darker, but its area did not extend. I was very reluctant to amputate on account of the patient's general feebleness and the absence of a pulse in the right femoral artery. seemed likely that gangrene of the flaps would follow even a high amputation. On Jan. 14th discharge began to come from between the first and second toes, and the tissues over the fifth metatarsal showed signs of moist gangrene. All the toes were now quite black and shrivelled. The temperathe toes were now quite black and shrive to $100^{\circ}$ and pain increased.

I considered, as an alternative to amputation, Leriche's operation of sympathectomy, but the patient's pipe-like femoral artery seemed unpromising material for such a delicate operation as the excision of a sleeve of tunica adventitia. It occurred to me that the object of the operation could be secured in a much simpler way by injecting the outer coat of the artery with alcohol. This is, of course, tho present method of choice for destroying the conductivity of sensory nerves, and there seemed no reason to doubt that it would be equally effective for the sympathetic nerves. I feared, groundlessly as it turned out, that the arterial narrowing was entirely due to organic thickening of the walls, and would be uraffected by artificial vaso-motor paralysis, but nevertheless, as amputation was the only alternative, I decided to try the alcohol injection.

On the 23rd, under light ether anæsthesia, I exposed the right femoral artery in IIunter's canal, divided its sheath, and with a very fine hypodermic needle, obliquely introduced in to the tunica adventitia, I injected about $1 \mathrm{minims}$ of rectified spirit at each of four equidistant points around its circumference. No pulsation could be felt in the artery, even when exposed. No local change in the calibre of the artery followed the injection, but where the alcohol was injected a whitish, ill-defined, opaque band appeared around the vessel. The wound was irrigated with flavine and closed. The following day, the 24 th, the patient volunteered the statement that she felt better and more comfortable in her foot. During the few days previous there had been increas. ing pain in the foot. On examination the foot, contrary to all previous observations, was decidedly warmer than the sound foot. A return of warmth was noticeable even on the dark, purplish-black areas on the dorsum and sole.

On the 31st an area as large as a penny on the dorsum at the base of the great toe, which had been black, was found to have recovered its normal colour. Surface thermometers were used for the first time. They showed on the dorsum of the right (affected) foot above the gangrenous area a temperature of $31^{\circ} \mathrm{C}$. At the corresponding spot on the left foot the temperature was $29^{\circ} \mathrm{C}$.

On Feb. 15th the right leg and foot were still decidedly warmer than the left. The dark purple area on the dorsum was becoming lighter, and islets of comparatively normal colour were appearing over the discoloured area on the sole. The foot was warm up to the base of the toes. On Feb. 17th the surface temperatures were, right foot $31.5^{\circ}$, left foot $27^{\circ}$. On the 21 st right foot $31.75^{\circ}$, left foot $27^{\circ}$. There was thus a slowly progressive increase in the surface temperature of the right foot, accompanied by a fall in the temperature of the left (normal) foot. The latter event may perhaps be accounted for by the diversion to the right lower limb, owing to the relaxation of its arteries, of an undue share of blood. The operation was in fact robbing Peter, the left foot, to pay Paul, the gangrenous foot. The condition of the left foot, which was distinctly more cold and blue than before, caused me some anxiety, and a prophylactic injection of the left femoral artery was considered, but was postponed in view of the predominant needs of "Paul" at the moment. By March 27 th, when the second pair of drawings were done, the dorsum had recovered its colour as far as and a little way along the toes. Definite inflamed lines of demarcation had appeared round each toe near its base. There was still some light mottled discoloration of the sole of the foot, but it was rapidly clearing up. There appears to be every prospect that amputation will be avoided and that the patient will recover with the loss of her toes only.

By the 31st the line of demarcation round the toes had become a deep groove and the dead portions of the toes could be rocked upon the still living portions. The excellence of the vascular supply right up to the line of demarcations was shown not only by the bright colour of the granulations forming the floor of the groove, but by the entire absence of sepsis at the line of separation.

In this case the revitalisation of a portion of the foot which had shown all the early signs of mortification and had been provisionally marked off for separation by a slight but definite line of demarcation, is a phenomenon unique in my experience. Was this tissue dead, and did it undergo a local resurrection? It seems more likely that, though it showed the signs of death, it was in a moribund but still recoverable condition when the reviving tide of blood reached it.

Case 2.-A male, aged 76, was admitted to the Middlesex Hospital under my care on March 7 th, 1922. There was nothing important in his history except that he had suffered from painter's colic severely at the age of 45 years. Six weeks before admission one evening the patient was bathing his feet, when suddenly his left foot became cold and numb. He rubbed his left foot but could not get it warm. There was pain in the foot on trying to walk. He went to bed and had his left foot rubbed for half an hour and painted with iodine. During the night the pain became more acute, and medical advice was sought. During the succeeding three weeks the pain was acute, but it has gradually diminished, 